\title{
Association between Porphyromonas gingivalis and tumor-promoting status in esophageal squamous cell carcinoma
}

Miao-Fen Chen ( $\nabla$ miaofen@adm.cgmh.org.tw)

Chiayi Chang Gung Memorial Hospital

Ming-Shian Lu

Chiayi Chang Gung Memorial Hospital

Ching-Chuan Hsieh

Chiayi Chang Gung Memorial Hospital

Wen-Cheng Chen

Chiayi Chang Gung Memorial Hospital

\section{Research}

Keywords: PG, Calcitriol; IL-6; esophageal SCC

Posted Date: April 20th, 2020

DOI: https://doi.org/10.21203/rs.3.rs-22376/v1

License: (c) (i) This work is licensed under a Creative Commons Attribution 4.0 International License.

Read Full License 


\section{Abstract}

Background The microbiota is reported to affect tumor growth and the microenvironment of gastrointestinal cancers. However, the possible role of keystone bacterial infection in the development of esophageal squamous cell carcinoma (EsoSCC) remains unclear. In the present study, we studied the relationship between the oral microbiome, the periodontal pathogen Porphyromonas gingivalis (PG), and EsoSCC and elucidated the underlying mechanisms.

Methods The microbiota in oral biofilms from patients was studied to identify bacterial biomarkers associated with EsoSCC by bioinformatics analyses. We then performed immunohistochemistry to examine the presence of PG in the esophageal tissues and analyze its relationship with the clinicopathologic characteristics in 156 EsoSCC patients. Furthermore, we investigated the role of PG infection in the progression of EsoSCC and its relationship with tumor microenvironment.

Results The microbiota profiles from oral biofilms revealed that the abundance of PG was associated with a higher risk of developing EsoSCC. The presence of PG was detected immunohistochemically in $57 \%$ of cancerous tissues and was associated with a higher clinical stage and poor prognosis. Using cellular experiments and a 4-nitroquinoline 1-oxide (4NQO)-induced tumor animal model, the presence of PG was found to be associated with a higher risk of esophageal cancer. Increased IL- 6 expression associated with an augmented epithelial-mesenchymal transition and recruitment of myeloid-derived suppressor cells were possible underlying mechanisms. Furthermore, in animal model including 4NQOinduced tumor and xenograft tumor model, we showed that the increased vitamin $D_{3}$ induced by UVB light treatment obviously down-regulated IL-6 signaling and subsequently attenuated the increased risk of esophageal cancer induced by PG infection.

Conclusions Our findings suggested that the presence of PG played an important role in the progression of esophageal cancer. Directly targeting PG or increasing vitamin $\mathrm{D}_{3}$ could be a promising strategy for the treatment of EsoSCC patients with PG infection noted by oral microbiota screening.

\section{Background}

Esophageal cancer is a common gastrointestinal tumor, and difficult to cure [1]. Despite advances in medicine, the lack of obvious symptoms has led to more esophageal cancer patients being diagnosed at more advanced stages, and these advanced stages are associated with reduced survival [2]. Therefore, to identify the potential molecular markers and understand the molecular mechanisms underlying tumor aggressiveness is important for esophageal cancer.

There is mounting evidence that microorganisms are directly linked to an fifth of cancers [3]. Microbes present at mucosal sites can affect cancer growth and spread $[3,4]$, and dysbiosis is involved in the progression of aerodigestive tract malignancies $[5,6]$. Gut microorganisms are crucially involved in tumor-promoting inflammation $[6,7]$. The presence of specific species within the microbiota was reported to positively correlate with the clinical outcome of esophageal cancers $[8,9]$. Adenocarcinoma and 
squamous cell carcinoma are the predominant histologic types of esophageal cancer. Although the prognosis with either type of esophageal cancer is poor, the clinical and molecular features between adenocarcinoma and squamous cell carcinoma are different [10]. The relationship between the microbiome and esophageal adenocarcinoma development has been experimentally investigated $[5,11]$. The microbiome is less well characterized in esophageal SCC (EsoSCC). In addition, it has been reported that the oral microbiota shapes the esophageal microbiome and may contribute to esophageal carcinogenesis $[5,11,12]$. To study the association between the oral microbiome and the risk of EsoSCC may be able to develop a novel research paradigm as a screening tool. A better understanding of the role of oral microbiome and the keystone pathogen in EsoSCC could have clinical implications. Therefore, we examined the microbiome composition in oral and esophageal specimens and elucidated the mechanism(s) by which the microbiome affects tumor behavior in EsoSCC.

\section{Materials And Methods}

\section{Patient characteristics}

This present study was approved by the Institutional Review Board of our hospital. We enrolled 156 EsoSCC patients who received curative treatment according to the guidelines proposed by the oncology team at our hospital. The specimens collected from these patients at diagnosis were subjected to immunochemical analysis. We also analyzed patient demographic, diagnosis, clinical stage, and treatment data (Table 1). In addition, oral biofilms were obtained from 26 patients with EsoSCC and 9 healthy donors.

\section{Immunohistochemical staining (IHC)}

Briefly, formalin-fixed, paraffin-embedded tissues from EsoSCC at diagnosis were cut into 4- $\mu \mathrm{m}$ sections, and were subjected to $\mathrm{IHC}$ staining. The slides were incubated overnight at $4^{\circ} \mathrm{C}$ with antibodies against IL6 and PG (1:100). In addition, there were esophageal cancer specimens from 21 EsoSCC patients were converted into tissue microarray (TMA) blocks, which contained cancer specimen and the adjacent nonmalignant epithelium.

\section{Microbiome assay}

We extracted DNA from oral biofilm samples. The detail was described in Suppl. Method. Sequencing of the multiplexed pooled libraries was performed on a MiSeq system (Illumina, USA). The quality-filtered reads (from $\mathrm{N}=35$ samples) were clustered into operational taxonomic units (OTUs) against Ribosomal Database Project (RDP) framework 11.5, and assigned taxonomy using QIIME V1.8.0. The a-diversityand the $\beta$-diversity was assessed at the OTU level using unweighted and weighted UniFrac distances. The differential abundances of OTUs between healthy controls and patients with EsoSCC were determined using linear discriminant analysis effect size (LEfSe).

\section{Cell and bacterial cultures}


Human EsoSCC cell lines, including CE81T and TE2 cells, were cultured. Porphyromonas gingivalis (PG) (strain ATCC 33277) was grown in GAM broth (Nissui, Tokyo, Japan). The EsoSCC cells were infected with PG at a multiplicity of infection (MOI) of $1: 10$ for $24 \mathrm{~h}$ or $72 \mathrm{~h}$ at $37^{\circ} \mathrm{C}$. Following PG infection or control (the same media in the absence of $P G)$, these cells were washed for following in vitro experiments.

\section{Animals and experimental Design}

The Experimental Animal Ethics Committee of our hospital approved this protocol. Six-week-old C57BL/6 mice were used for the 4NQO-induced tumor model $[13,14]$.

Briefly, the mice were allowed access to the drinking water containing 4NQO at a concentration of 100 $\mu \mathrm{g} / \mathrm{ml}$ (tumor-induced group) or the solvent only (control group) at all times for 16 weeks and analyzed esophageal lesions at 12-14 weeks $[14,15]$. In the tumor-induced group, mice were randomly divided into the 4NQO group, 4NQO+PG group, 4NQO+light group, and 4NQO+PG+light group. The animals in the 4NQO+PG group and 4NQO+PG+light group were repeatedly infected with Porphyromonas gingivalis (PG) $\left(200 \mu \mathrm{L}\right.$ of $10^{10}$ bacteria/mL) [16] (three times/week) for 2 weeks prior to 4NQO administration and for another 10 weeks (twice a week) after 8 weeks of 4 NQO treatment. Because ultraviolet B light-emitting diodes (UVB-LEDs) have been reported to be efficient and effective in producing vitamin $\mathrm{D}_{3}\left(1 \mathrm{a}, 25(\mathrm{OH})_{2}\right.$ $\mathrm{D}_{3}$; calcitriol) [17], we examined the role of VitD 3 in vivo using UVB light treatment. UVB-LEDs of $300 \mathrm{~nm}$, obtained from the Instrument Technology Research Institute (Hsinchu, Taiwan), were used in this study. In mice that received light treatment (the 4NQO+light and 4NQO+PG+light groups), UVB light treatment (30 minutes a day; 5 times per week) was started 12 weeks after the initiation of 4-NQO treatment. In addition, athymic mice were used for xenograft esophageal tumor models. In the orthotopic tumor model, human esophageal cancer cells $\left(1 \times 10^{6}\right.$ cells per implantation) were intraoperatively injected into the wall of the esophagus. The extent of orthotopic tumor growth was measured at 3 weeks after implantation.

\section{Small-animal imaging of tumors}

In vivo optical imaging of mice for tumor model was performed with fluorescence molecular tomography (FMT) to detect the glucose-uptake-signals by DG750 for observation of tumor growth at different time points. DG750, a fluorescent probe for in vivo imaging of tumors, exhibit an elevated glucose uptake rate in tumor compared to surrounding tissues. After imaging examination, gross and tissue examinations were performed to evaluate the esophageal lesions in mice.

\section{Flow cytometric analysis (FACS) of myeloid-derived suppressor cells (MDSCs)}

Current data suggest that MDSCs are a group of phenotypically heterogeneous myeloid cells that have common biological activities. In the present study, FACS was carried out on single-cell suspensions prepared from murine spleen after digestion and immunostaining for Gr1 and CD11b, as described previously [15]. 
Immunofluorescence (IF) staining \& Enzyme-linked immunosorbent assay (ELISA) of in vitro and in vivo IL-6 and vitamin $D_{3}$ levels

The detail was described in Suppl. Methods.

\section{Results}

\section{Oral microbiota in EsoSCC}

To characterize the oral microbiome community in EsoSCC, oral biofilms over dental surfaces were collected from EsoSCC patients and controls. As shown in Suppl. Figure 1a-b, ESCC samples differed from controls in oral microbiome a-diversity, (species richness) and $\beta$-diversity (microbiome composition). There was a significant difference in the overall oral microbiome composition between the cancer and control groups $(p=0.001)$, as measured by Adonis analysis. To identify the differentially enriched species within groups, the LEfSe method was used. Streptococcus mutans, Veillonella parvula, Streptococcus peroris, and P. gingivalis (PG) were abundant in the oral biofilms from EsoSCC samples (Figure 1a). To further examine if $P G$ is more frequent in esophageal cancer tissue, we employed qPCR to examine the presence of PG 16S rDNA in fresh esophageal specimens. The data revealed that PG16S rDNA were more frequent detected in EsoSCC specimen than non-malignant specimen (Figure 1b).

\section{Positive PG staining correlates with the prognosis of EsoSCC}

Figure 1c shows representative positive and negative stained slides for PG in human EsoSCC tissue specimens at diagnosis. The IHC data for TMA slides from 21 EsoSCC specimens revealed that PG was detected more frequently in $12(57 \%)$ cancerous tissues than in adjacent esophageal mucosa $(24 \%)$. Since an association between PG infection and EsoSCC was noted, we examined whether positive staining with anti-PG antibodies was associated with the prognosis of EsoSCC. The clinical characteristics of the EsoSCC patients are presented in Table 1. For the total 156 EsoSCC specimens, 89 (57\%) showed the presence of PG. Positive staining for PG was positively related to poor differentiation and advanced stage in EsoSCC patients $(p<0.05)$. Moreover, the presence of PG was significantly associated with a higher disease failure rate (developing locoregional recurrence and distant metastasis) and reduced overall survival rate (Figure1d \& Suppl. Fig 2). These findings suggested that the presence of PG contributes to tumor aggressiveness and poor prognosis in EsoSCC.

\section{PG infection-associated esophageal tumorigenesis in 4NQO-induced tumor model}

We established and characterized an experimental mouse model featuring 4NQO-induced esophageal cancer and PG infection, as described in the Methods. At 12 14 weeks after the 16-week 4NQO treatment, the FMT images were taken, and then the mice were analyzed, and the esophagus was removed for further evaluation. Figure 2a demonstrated the lesions on the esophagus in 4NQO-treated mice, and the pathological esophageal lesions including hyperplasia/papilloma, carcinoma in situ (CIS), and invasive carcinoma. The validity of FMT at 12 weeks after the 16-week 4 NQO treatment for detecting esophageal 
lesions in mice is shown in Figure 2b. Furthermore, esophageal carcinoma (CA) including carcinoma in situ (CIS) and invasive carcinoma, had a significantly higher glucose-uptake signal than those with benign tumor (hyperplasia and papilloma). We also examined the link between tumor progression and MDSC recruitment in mice. There were significantly higher MDSCs in mice with CA (Figure 2c). In addition, PG infection was associated with the increases in glucose-uptake signal by FMT and MDSC recruitment by FACS in 4NQO-treated mice (Figure 2d-e).

\section{PG enhanced tumor invasion}

To examine whether the invasiveness of cancer cells was modulated by PG, we performed an in vitro invasion assay to analyze the invasiveness of PG-infected EsoSCC cells. Figure 3a shows that CE81T and TE2 cells coincubated with PG had a prominent increase in invasive potential compared with control cells. Epithelial-mesenchymal transition (EMT) play a critical role in the invasiveness of cancer [18], and we examined whether it is the underlying mechanism responsible for the enhanced tumor aggressiveness induced by PG infection. As shown in Figure 3b, PG-infected EsoSCC cells had augmented EMT changes and presented with increased $\beta$-catenin and matrix metalloproteinase (MMP)-9 expression and decreased E-cadherin expression. In addition, cancer stem cells (CSCs) were reported to be key event in aggressive tumor behavior [19], including upper aerodigestive tract cancer. As shown in Figure 3c-d \& Suppl. Figure 3, coincubation with PG significantly stimulated the expression of CD44 and ALDH1 which are CSC markers in human esophageal cancer cells.

\section{The correlation between PG infection and IL-6 signaling}

We previously reported that overexpressed IL-6 correlated with the poor prognosis of EsoSCC, and activation of the IL-6/STAT3 pathway plays a critical role in CSC formation and EMT [20, 21]. Furthermore, activated IL-6/STAT3 signaling has been shown to be the adaptive pathway contributing to microbiota-induced tumor progression [3]. Accordingly, we investigated the correlation between PG and IL6 signaling. Figure 4a-b shows that PG significantly increased IL- 6 expression in cancer cells and the supernatant in cell cultures. Furthermore, a significantly positive correlation was found between cancer specimens that expressed IL-6 and the presence of PG (Fig. 4c \& Table 1). It has been reported that bacterial infection-related autophagy may have an effect on tumor initiation and cancer treatment $[3,22]$. PG is reported to activate cellular autophagy and stimulate pro-inflammatory cytokines [23]. As shown in Figure 4d-e \& Suppl. Figure 4, the PG-infected cells had increased autophagy, represented by the conversion of LC3-I to LC3-II, associated with increased IL-6 expression. Furthermore, pre-treatment with the autophagy inhibitor 3-MA $10 \mathrm{mM}$ for $1 \mathrm{~h}$ attenuated the expression of IL-6 in PG-infected cells. The results reveal that autophagy may be one of the mechanisms contributing to the increased IL- 6 noted in PG-infected cancer cells.

\section{Effect of calcitriol on the invasiveness of tumors with PG infection}

In the gut, calcitriol was reported to have anti-inflammatory and anti-infectious effects in experimental models [24]. We previously reported that calcitriol abrogated IL-6-induced tumor aggressiveness and 
inhibit the promotion of esophageal tumor. Accordingly, we examined whether calcitriol attenuates the tumor-promoting status induced by PG infection. As demonstrated in Figure 5a, calcitriol inhibited the PGinduced increase in IL- 6 and activated STAT3 in esophageal cancer cells. In vitro, incubation with calcitriol for $48 \mathrm{~h}$ attenuated the invasion ability in association with reversing the expressions of $\beta$ catenin, MMP-9 and ALDH1 induced by PG infection (Figure 5b-d \& Suppl. Figure 5). Based on the data, we suggest that calcitriol reverses EMT and CSC changes and subsequently attenuates tumor invasiveness in PG-infected esophageal cancer, which is mediated by regulating IL- 6 signaling, at least a part.

\section{Increased calcitriol induction by UVB light reverses the tumor-promoting status induced by PG infection}

The concentrations of calcitriol required for antineoplastic effects are usually too high to achievable safely in patients with vitamin $D_{3}$ is dosed daily. Therefore, we examined the effect of increased calcitriol induced by UVB irradiation with a $300 \mathrm{~nm}$ LED. As shown in Figure 6a, after UVB irradiation, serum calcitriol increased markedly in mice. To investigate whether UVB light treatment ameliorates the induction of esophageal cancer, mice were irradiated per a chronic exposure protocol. As shown in Figure $6 b$ \& Suppl. Figure 6a-b, UVB light treatment increased calcitriol in serum, with decreased IL-6 in serum, attenuated MDSC recruitment and a lower incidence of esophageal CA formation. Moreover, an orthotopic tumor implantation model was used to examine the effect of UVB light treatment on xenograft tumor growth. As demonstrated in Figure 6c-e, the UVB light treatment decreased the glucose-uptake signals, decreased tumor size and attenuated EMT change.

\section{Discussion}

The gut microbiome has been shown to play a crucial role in diseases, including some cancers $[3,4]$, and influence tumor development and progression in the gastrointestinal tract by experimental evidence. The esophageal microbiome is reported to be similar to the oral microbiome in composition, but with key taxonomic differences $[12,25]$. Poor oral hygiene and periodontal disease are reported to increase esophageal cancer risk [26]. In our country, greater than $90 \%$ of esophageal cancers are EsoSCC. Accordingly, we examined the relationship between the oral microbiome and EsoSCC. with the oral microbiota in EsoSCC and control samples differed in terms of microbiome composition ( $\beta$-diversity) and species richness (a-diversity). By LDA, we identified several oral bacterial species associated with EsoSCC risk that reached the significance threshold. A causal relationship between specific bacterial infections and the development of certain malignancies has been suggested. Altered bacterial microbiota and the abundance of the periodontal pathogen PG in oral samples are reported to be associated with a higher risk of EsoSCC $[25,27]$. Similarly, our microbiota analysis showed that the presence of PG in dental biofilm samples was more likely in EsoSCC patients than controls. Furthermore, we found that PG $16 \mathrm{~S}$ rRNA was more frequently detected in EsoSCC specimens than in nonmalignant tissues by real-time PCR. PG, a key oral pathogen, is reported to invade epithelial cells and modulate host immune responses. PGmediated protumorigenic mechanisms, such as immune evasion and carcinogenesis, have been posited $[28,29]$. Since an association between PG infection and EsoSCC was proposed, we examined whether PG 
positivity, as assessed by staining with anti-PG antibodies, is associated with the progression of esophageal cancer. According to the IHC data, 89 (57\%) patients showed the presence of PG, and the positivity of PG was related to poor differentiation, advanced stage, high disease failure rate and reduced overall survival rate. These findings suggested that the presence of PG contributes to tumor aggressiveness and poor prognosis in EsoSCC. To further investigate the link between PG infection and esophageal tumor promotion, we examined the effect of $P G$ on tumor promotion in an immunocompetent host: the 4NQO-induced esophageal-tumor model. In 4NQO-treated mice, PG infection was associated with elevated glucose-uptake signal in the esophageal lesions and an increased incidence of developing esophageal CA. The process of EMT and CSCs appears to be relevant for the invasiveness of epithelial tumors, including esophageal cancer $[18,30]$. In cellular experiments, coincubation with PG augmented EMT changes and the expression of CSC markers in esophageal cancer cells. Therefore, we suggest that the presence of PG plays a role in the induction of esophageal tumors.

There were several potential mechanisms through which the microbiome participates in carcinogenesis $[4,7]$, including chronic inflammation. It has recently become apparent that the host inflammatory response links the presence of a pathogen to cancer development, and gut microorganisms are crucially involved in tumor-promoting inflammation. Furthermore, activation of IL-6/STAT3 signaling have been shown contributing to microbiota-induced tumor progression $[3,31]$. In a previous report, we demonstrated that increased IL-6 was linked with poor prognosis in patients with EsoSCC [20, 21]. The induction of CSCs, augmented EMT and induction of an immunosuppressive tumor microenvironment were the responsible mechanisms. Accordingly, we examined the relationship between PG infection and IL-6 production in EsoSCC. In the 4-NQO tumor model, PG infection was associated with higher IL-6 expression in esophageal tissue and augmented recruitment of MDSCs. In addition, we demonstrated a positive correlation between PG and IL-6 staining in EsoSCC tumor specimens. PG, an important periodontal pathogen, has been reported to activate cellular autophagy, a possible mechanism in inflammatory disease. Multiple groups have observed that the secretion of IL 6 depends upon autophagy in some cancers [32,33]. In vitro, when esophageal cancer cells were invaded by PG, autophagy was increased, as was IL-6 production. Furthermore, the recruitment of MDSCs allows cancer cells to escape from host immune systems [34]. We showed that PG infection modulated the local tumor microenvironment in association with increased MDSC recruitment in EsoSCC. Conversely, blocking IL-6 abrogated the induction of EMT and CSC changes in cancer cells with PG infection. Based on these findings, we suggest that PG promotes esophageal tumor growth and that IL-6-mediated tumor promotion and immune modulation play critical roles in this process.

It has been reported that vitamin $D$ has the potential role in modulating host immune responses, cancer prevention, and affecting the gut microbiome [24,35]. We previously reported that calcitriol abrogated IL6-induced aggressive tumor behavior and MDSC recruitment to inhibit esophageal tumor promotion. In the present study, we demonstrated that calcitriol reversed the invasion ability associated with EMT and the CSC changes induced by PG in vitro. Calcitriol could be obtained from either dietary sources or production by exposure to sunlight. The concentrations of calcitriol required for antineoplastic effects are usually supraphysiological and may induce predictable hypercalcemia. Therefore, in the present study, we 
applied UVB light treatment to increase serum vitamin $\mathrm{D}_{3}$ levels. Our results demonstrate that in $\mathrm{C} 57 \mathrm{BL} / 6$ mice, UVB irradiation for 30 minutes for 5 days per week significantly increased calcitriol in serum in association with lower glucose uptake in esophageal lesions and a decreased risk of developing esophageal CA. In addition, lower IL-6 levels and attenuated MDSC recruitment were noted in the UVBirradiated group. Therefore, we suggest that calcitriol supplementation from dietary sources or UVB exposure could reverse the tumor-promoting status induced by PG infection and should be a promising strategy for the prevention and treatment of EsoSCC for patients with PG infection noted by oral microbiota screening.

\section{Conclusion}

We suggested that the presence of PG played an important role in the promotion of esophageal cancer. The IL-6-mediated tumor-promoting status is the underlying mechanism responsible to tumor aggressiveness. Furthermore, good oral hygiene to decrease PG infection and appropriate sunbathing to increase vitaminD3 might be a promising strategy to aid the treatment of PG-positive EsoSCC.

\section{Abbreviations}

EsoSCC: Esophageal squamous cell carcinoma; PG: Porphyromonas gingivalis;

4NQO: 4-nitroquinoline 1-oxide; OUTs: operational taxonomic units;

UVB: Ultraviolet B; LED: Light-emitting diodes;

Calcitriol: Vitamin D3 (1a, 25(OH)2 D3

FMT: Fluorescence molecular tomography; FACS: Flow cytometric analysis;

MDSCs: Myeloid-derived suppressor cells; IF: Immunofluorescence;

ELISA: Enzyme-linked immunosorbent assay; CA: carcinoma;

CIS: Carcinoma in situ; EMT: Epithelial-mesenchymal transition;

CSCs: Cancer stem cells; IHC: Immunohistochemical staining;

\section{Declarations}

\section{Ethics approval and consent to participate}

The Institutional Review Board of Chang Gung Memorial Hospital approved the human study (No. 201801600B0). The written consents were signed by the patients for their specimen and information to be stored in the hospital and used for research. 
The animal protocol was approved by the Committee on the Ethics of Animal Experiments of Chang Gung Memorial Hospital (No. 2018092510). This animal study was performed in strict accordance with the recommendations in the Guide for the Care and Use of Laboratory Animals as promulgated by the Institutes of Laboratory Animal Resources, National Research Council, USA.

\section{Consent for publication}

Not applicable.

\section{Availability of data and materials}

Not applicable.

\section{Competing interests}

The authors declare that they have no competing interests.

\section{Funding}

The work was support by Chang Gung Memorial Hospital. Grant CMRPG6H0561-2 (to M.F. Chen)

\section{Authors' contributions}

Conception and design: MFC, MSL; Development of methodology: MFC, $\mathrm{CCH}$; Acquisition of data (provided animals, acquired and managed patients): MFC, MSL, CCH, WCC; Analysis and interpretation of data: MFC, CCH; Writing, review, and/or revision of the manuscript: MFC, MSL, WCC; Study supervision: MFC.

All authors read and approved the final manuscript

\section{Acknowledgments}

We would like to thank Chih-Ming Hsu for technical assistance

\section{References}

1. Enzinger PC, Mayer RJ. Esophageal cancer. N Engl J Med 2003, 349:2241-2252.

2. Pottgen $C$, Stuschke M. Radiotherapy versus surgery within multimodality protocols for esophageal cancer-a meta-analysis of the randomized trials. Cancer Treat Rev 2012, 38:599-604.

3. Garrett WS. Cancer and the microbiota. Science 2015, 348:80-86.

4. Dzutsev A, Goldszmid RS, Viaud S, et al. The role of the microbiota in inflammation, carcinogenesis, and cancer therapy. Eur J Immunol 2015, 45:17-31.

5. Baba Y, Iwatsuki M, Yoshida N, et al. Review of the gut microbiome and esophageal cancer: Pathogenesis and potential clinical implications. Ann Gastroenterol Surg 2017, 1:99-104. 
6. Nasrollahzadeh D, Malekzadeh R, Ploner A, et al. Variations of gastric corpus microbiota are associated with early esophageal squamous cell carcinoma and squamous dysplasia. Sci Rep 2015, 5:8820.

7. Elinav E, Nowarski R, Thaiss CA, et al.Inflammation-induced cancer: crosstalk between tumours, immune cells and microorganisms. Nat Rev Cancer 2013, 13:759-771.

8. Gao S, Li S, Ma Z, Liang S, et al. Presence of Porphyromonas gingivalis in esophagus and its association with the clinicopathological characteristics and survival in patients with esophageal cancer. Infect Agent Cancer 2016, 11:3.

9. Yamamura K, Baba Y, Nakagawa S, et al. Human Microbiome Fusobacterium Nucleatum in Esophageal Cancer Tissue Is Associated with Prognosis. Clin Cancer Res 2016, 22:5574-5581.

10. Siewert JR, Stein HJ, Feith M, et al. Histologic tumor type is an independent prognostic parameter in esophageal cancer: lessons from more than 1,000 consecutive resections at a single center in the Western world. Ann Surg 2001, 234:360-367; discussion 368-369.

11. Neto AG, Whitaker A, Pei Z. Microbiome and potential targets for chemoprevention of esophageal adenocarcinoma. Semin Oncol 2016, 43:86-96.

12. Norder Grusell E, Dahlen G, Ruth $M$, et al. Bacterial flora of the human oral cavity, and the upper and lower esophagus. Dis Esophagus 2013, 26:84-90.

13. Tang XH, Knudsen B, Bemis D, et al. Oral cavity and esophageal carcinogenesis modeled in carcinogen-treated mice. Clin Cancer Res 2004, 10:301-313.

14. Chen MF, Kuan FC, Yen TC, et al. IL-6-stimulated CD11b+ CD14+ HLA-DR- myeloid-derived suppressor cells, are associated with progression and poor prognosis in squamous cell carcinoma of the esophagus. Oncotarget 2014, 5:8716-8728.

15. Chen PT, Hsieh CC, Wu CT, et al. 1alpha,25-Dihydroxyvitamin D3 Inhibits Esophageal Squamous Cell Carcinoma Progression by Reducing IL6 Signaling. Mol Cancer Ther 2015, 14:1365-1375.

16. Wu JS, Zheng M, Zhang M, et al. Porphyromonas gingivalis Promotes 4-Nitroquinoline-1-OxideInduced Oral Carcinogenesis With an Alteration of Fatty Acid Metabolism. Front Microbio/2018, 9:2081.

17. Gorman S, Scott NM, Tan DH, et al. Acute erythemal ultraviolet radiation causes systemic immunosuppression in the absence of increased 25-hydroxyvitamin D3 levels in male mice. PLoS One 2012, 7:e46006.

18. McConkey DJ, Choi W, Marquis L, et al. Role of epithelial-to-mesenchymal transition (EMT) in drug sensitivity and metastasis in bladder cancer. Cancer Metastasis Rev 2009, 28:335-344.

19. Hermann PC, Bhaskar S, Cioffi M, et al. Cancer stem cells in solid tumors. Semin Cancer Biol 2010, 20:77-84.

20. Chen MF, Chen PT, Lu MS, et al. Role of ALDH1 in the prognosis of esophageal cancer and its relationship with tumor microenvironment. Mol Carcinog 2018, 57:78-88. 
21. Chen MF, Chen PT, Lu MS, et al. IL-6 expression predicts treatment response and outcome in squamous cell carcinoma of the esophagus. Mol Cancer 2013, 12:26.

22. Schwabe RF, Jobin C. The microbiome and cancer. Nat Rev Cancer 2013, 13:800-812.

23. Belanger $\mathrm{M}$, Rodrigues $\mathrm{PH}$, Dunn WA, et al. Autophagy: a highway for Porphyromonas gingivalis in endothelial cells. Autophagy 2006, 2:165-170.

24. Meeker S, Seamons A, Maggio-Price L, et al. Protective links between vitamin D, inflammatory bowel disease and colon cancer. World J Gastroenterol 2016, 22:933-948.

25. Peters BA, Wu J, Pei Z, et al. Oral Microbiome Composition Reflects Prospective Risk for Esophageal Cancers. Cancer Res 2017, 77:6777-6787.

26. Abnet CC, Kamangar F, Islami F, et al.Tooth loss and lack of regular oral hygiene are associated with higher risk of esophageal squamous cell carcinoma. Cancer Epidemiol Biomarkers Prev 2008, 17:3062-3068.

27. Chen X, Winckler B, Lu M, et al. Oral Microbiota and Risk for Esophageal Squamous Cell Carcinoma in a High-Risk Area of China. PLoS One 2015, 10:e0143603.

28. Inaba $\mathrm{H}$, Sugita $\mathrm{H}$, Kuboniwa $\mathrm{M}$, et al. Porphyromonas gingivalis promotes invasion of oral squamous cell carcinoma through induction of proMMP9 and its activation. Cell Microbio/2014, 16:131-145.

29. Whitmore SE, Lamont RJ. Oral bacteria and cancer. PLoS Pathog 2014, 10:e1003933.

30. Sampieri K, Fodde R. Cancer stem cells and metastasis. Semin Cancer Biol 2012, 22:187-193.

31. Yu H, Pardoll D, Jove R. STATs in cancer inflammation and immunity: a leading role for STAT3. Nat Rev Cancer 2009, 9:798-809.

32. New J, Thomas SM. Autophagy-dependent secretion: mechanism, factors secreted, and disease implications. Autophagy 2019, 15:1682-1693.

33. Maycotte P, Jones KL, Goodall ML, et al. Autophagy Supports Breast Cancer Stem Cell Maintenance by Regulating IL6 Secretion. Mol Cancer Res 2015, 13:651-658.

34. Schupp J, Krebs FK, Zimmer N, et al. Targeting myeloid cells in the tumor sustaining microenvironment. Cell Immunol 2019, 343:103713.

35. Cantorna MT, McDaniel K, Bora S, et al. Vitamin D, immune regulation, the microbiota, and inflammatory bowel disease. Exp Biol Med (Maywood) 2014, 239:1524-1530.

\section{Table}

Table 1 The Clinical characteristics of patients with ESCC 


\begin{tabular}{|c|c|c|c|}
\hline & \multicolumn{2}{|c|}{ No. of patients } & \multirow[b]{2}{*}{$p$ value } \\
\hline & IHC-PG(-) & IHC-PG(+) & \\
\hline Patients & 67 & 89 & \\
\hline \multicolumn{4}{|l|}{ Age } \\
\hline Range & $35.8 \sim 78.7$ & $37.3 \sim 78.5$ & 0.069 \\
\hline Median & 57.0 & 52.8 & \\
\hline \multicolumn{4}{|l|}{ Differentiation } \\
\hline $\mathrm{WD} \sim \mathrm{MD}$ & 41 & 34 & $0.004 *$ \\
\hline $\mathrm{PD}$ & 26 & 55 & \\
\hline Clinical stage & & & $0.004 *$ \\
\hline I-II & 22 & 12 & \\
\hline III-IV & 45 & 77 & \\
\hline LN metastasis & & & $0.035^{*}$ \\
\hline negative & 19 & 13 & \\
\hline positive & 48 & 76 & \\
\hline IL-6 staining & & & $<0.001 *$ \\
\hline negative & 46 & 34 & \\
\hline positive & 21 & 55 & \\
\hline \multicolumn{4}{|l|}{ Response to } \\
\hline Neoadjuvant Tx & & & $0.024 *$ \\
\hline Response & 54 & 57 & \\
\hline Non- response & 13 & 32 & \\
\hline $\begin{array}{l}\text { Surgery } s / p \\
\quad \text { Neoadjuvant Tx }\end{array}$ & & & 0.894 \\
\hline Yes & 21 & 27 & \\
\hline No & 46 & 62 & \\
\hline \multicolumn{4}{|c|}{ Local-regional Recurrence /persistent } \\
\hline & & & $0.022 *$ \\
\hline No & 38 & 34 & \\
\hline Yes & 29 & 55 & \\
\hline Distant metastasis & & & $0.028^{*}$ \\
\hline negative & 47 & 47 & \\
\hline positive & 20 & 42 & \\
\hline
\end{tabular}

Abbreviations: Neoadjuvant $\mathrm{Tx}=$ neoadjuvant chemoradiotherapy;

Figures 
PG

(a)

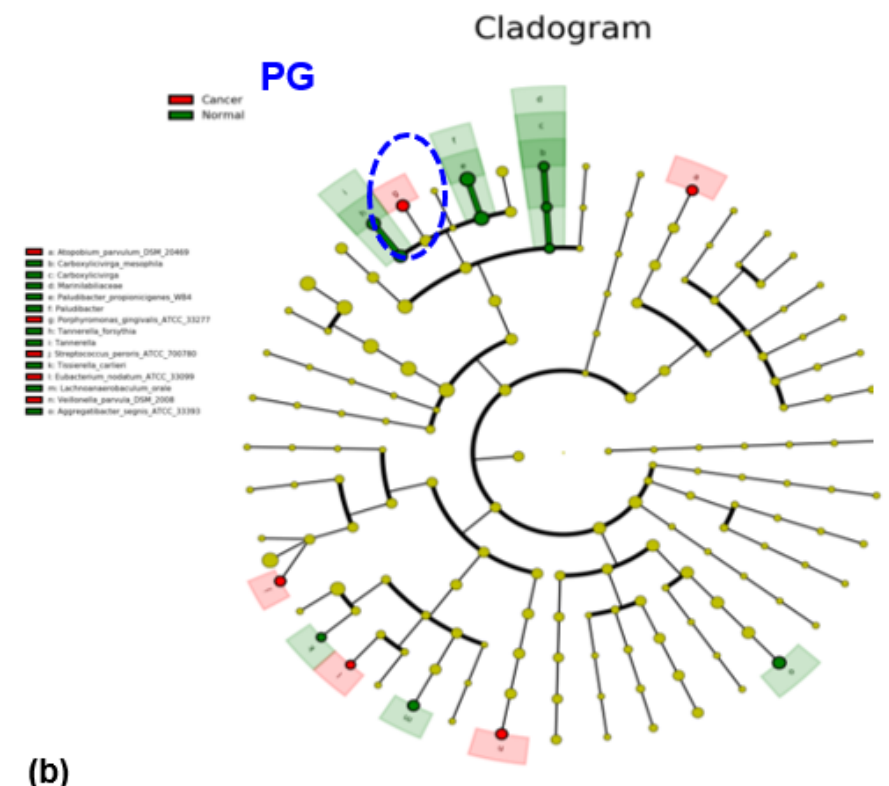

(b)

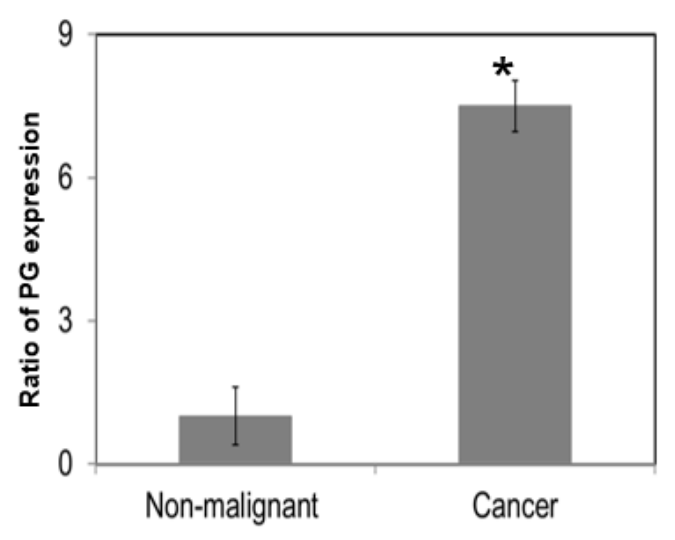

(c) Negative

Positive

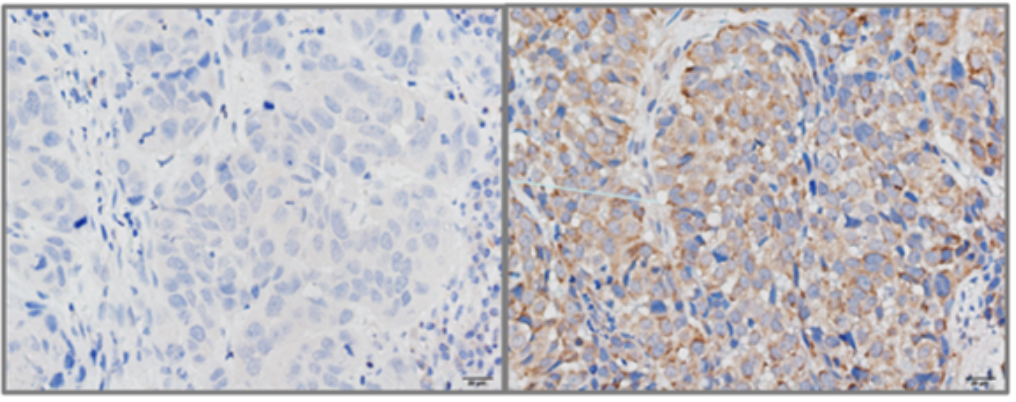

(d)

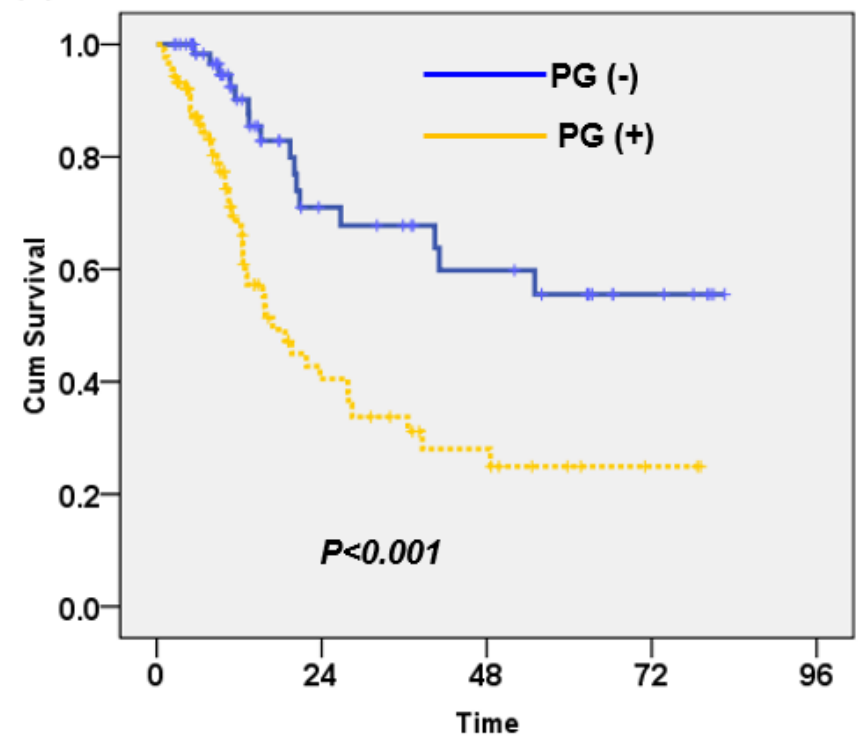

Figure 1

Relationship between the presence of PG and EsoSCC (a) Cladogram was applied to EsoSCC cases and matched controls using the oral microbiota analysis, and only species associated with EsoSCC are colored (Red: EsoSCC > control; Green EsoSCC < control); other species in networks are indicated by small outlined circles. (b) PCR-detected expression of PG in normal esophagus mucosa and cancerous tissues of EsoSCC. (c) Representative images of IHC staining with an anti-PG antibody of EsoSCC specimens are shown. (d) Survival differences demonstrated according to the presence of PG in overall survival. 


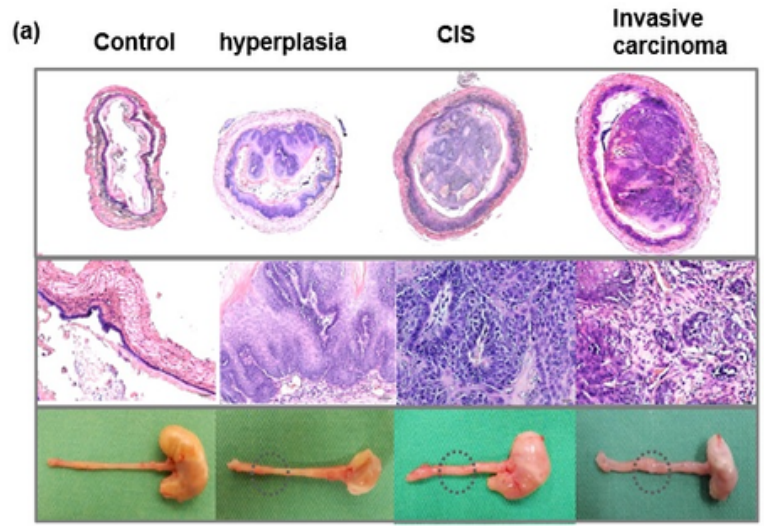

(b)

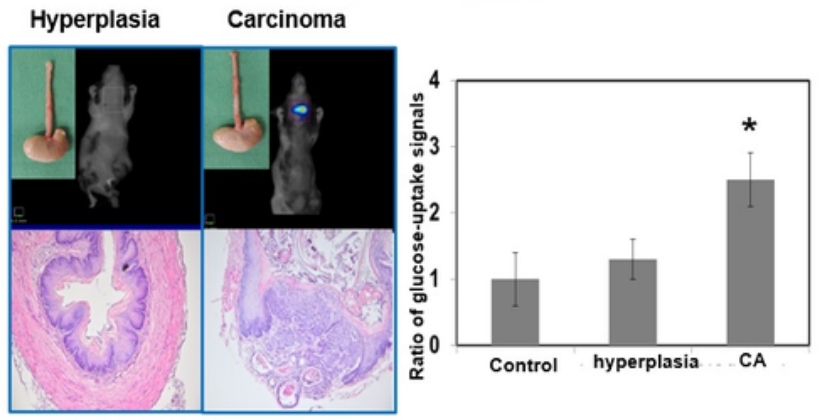

(c)
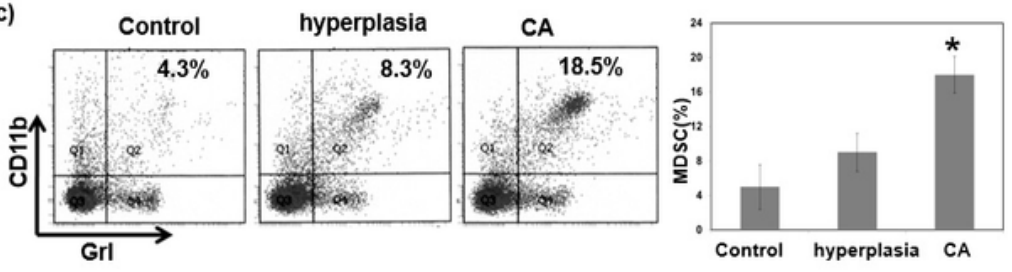

(d)
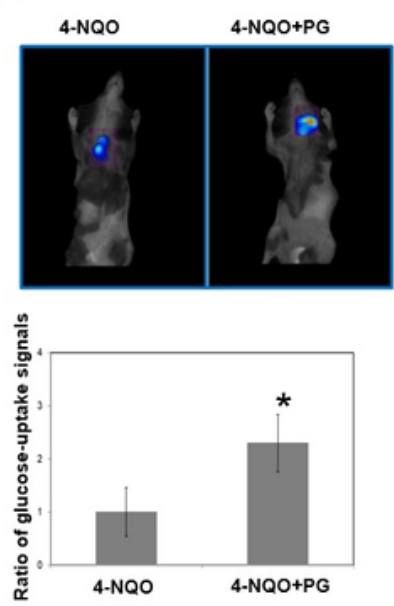

(e) Control 4-NQO 4-NQO+PG
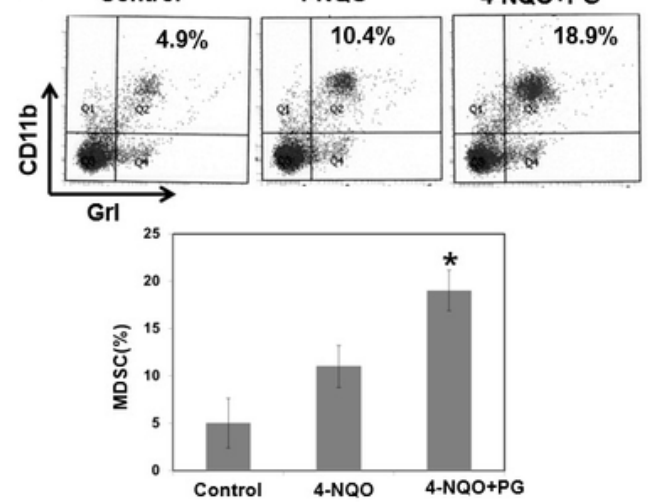

Figure 2

Effect of PG infection on tumor progression in 4NQO- tumor model (a) Images of the gross lesions and pathological findings of tissue sections from the animals treated with $4 \mathrm{NQO}$ or vehicle for 16 weeks that were followed for 12-14 weeks. Histological determinations included hyperplasia, CIS, and invasive carcinoma. (b) Representative FMT images from 4NQO-treated mice, and diagnosed with hyperplasia, CIS or invasive carcinoma. The glucose-uptake signals were calculated (y-axis shows the relative ratio, 
normalized to the signals under control conditions). Data points represent the means \pm SEMs. ${ }^{*}, p<0.05$. (c) Flow cytometric analysis of $C D 11 b+G r 1+M D S C$ from mice exhibiting hyperplasia or carcinoma (CA) after histological analysis. Representative images and quantitation are shown. The columns represent the means \pm SEM. ${ }^{*}, p<0.05$. The effects of PG infection on esophageal tumor formation in 4-NQO-treated mice were shown with representative images and quantitative data (d) FMT images with glucosee-uptake signals, and (e) Flow cytometric analysis of MDSC. Columns represent the means \pm SEM.

(a)

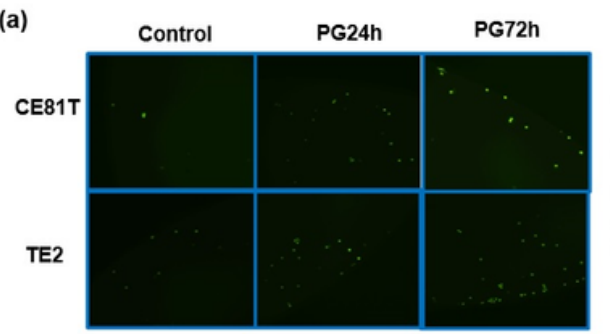

(b)

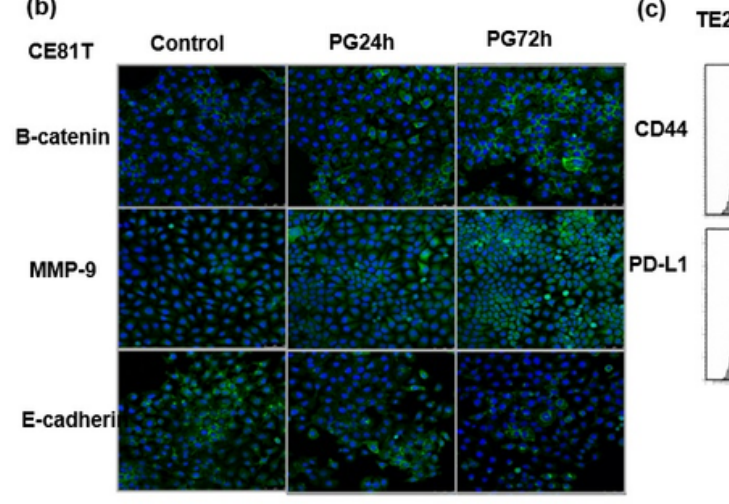

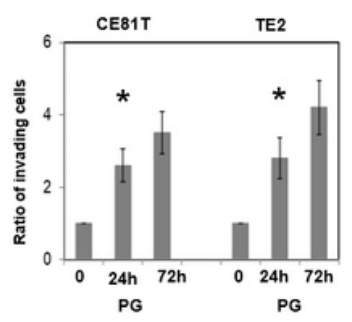

(c) TE2

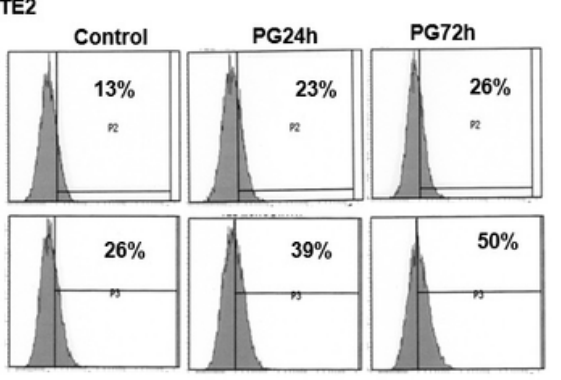

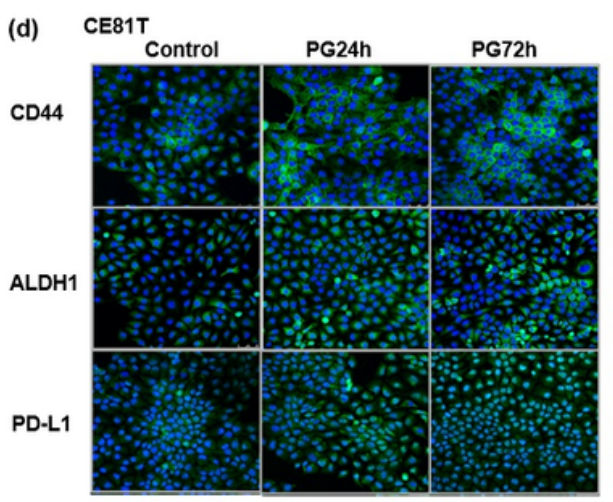

\section{Figure 3}

Effect of PG infection in tumor aggressiveness Effect of PG infection on (a) the invasive ability of esophageal cancer cells. Representative data and quantitative data (y-axis show the relative ratio, normalized to the number of invading control cancer cells) are shown; (b) EMT-associated proteins. The changes of E-cadherin, MMP-9 and B-catenin expression were evaluated by immunofluorescence in vitro. (DAPI, blue; target protein, green). Furthermore, effects of the presence of PG on CSC-related proteins with (c) Flow cytometric analysis of CD44 and PD-L1 using TE2 cancer cells; and (d) The changes of CD44, ALDH1 and PD-L1 expression were evaluated by immunofluorescence in vitro. (DAPI, blue; target protein, green) 

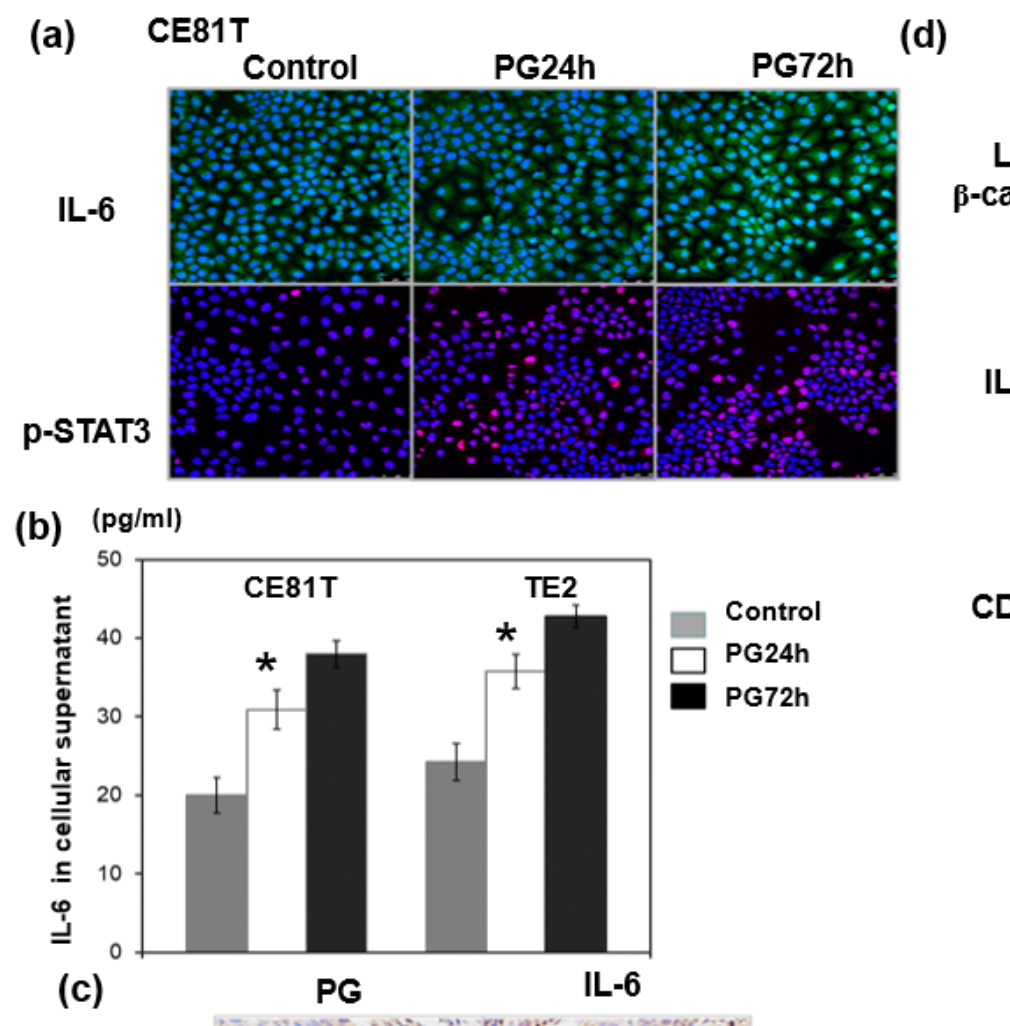

L-6

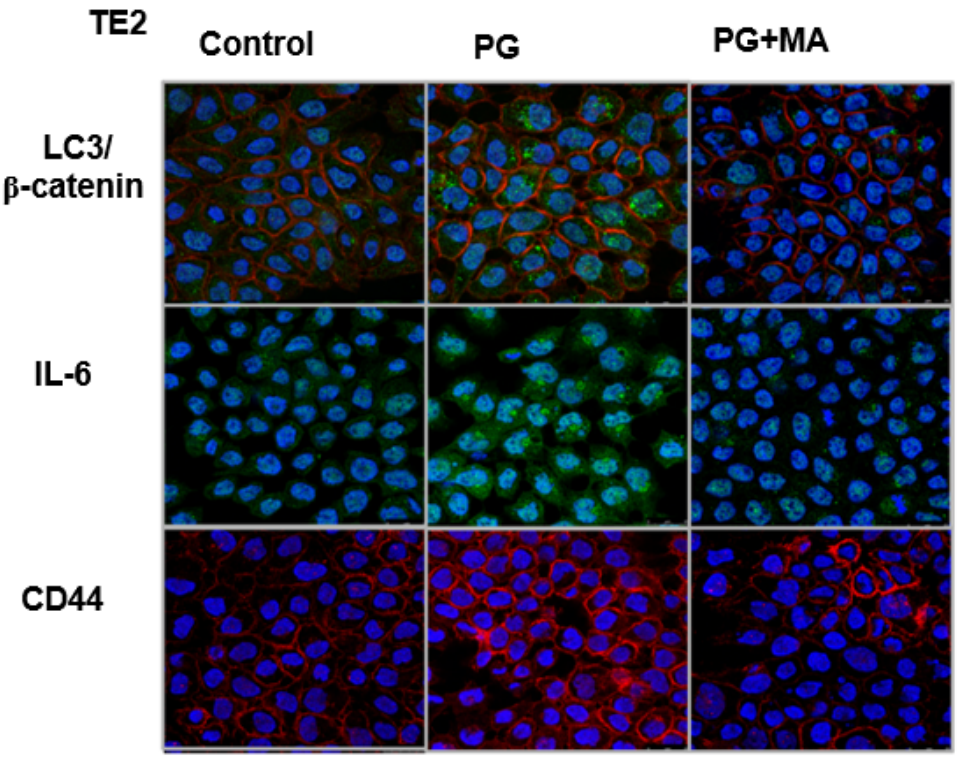

(e)

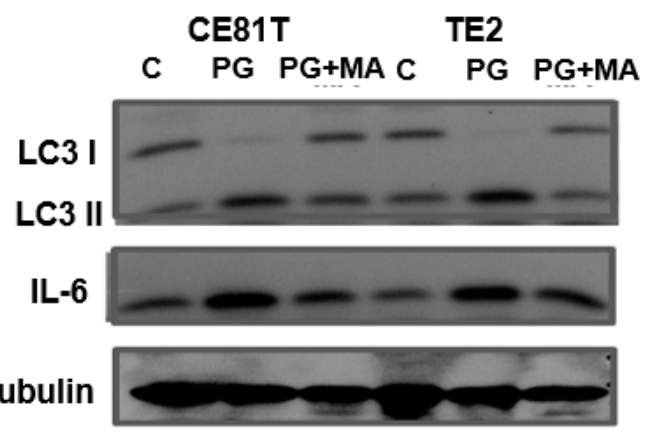

Negative

\section{Figure 4}

Effect of PG infection in tumor aggressiveness Effect of PG infection on (a) the invasive ability of esophageal cancer cells. Representative data and quantitative data (y-axis show the relative ratio, normalized to the number of invading control cancer cells) are shown; (b) EMT-associated proteins. The changes of E-cadherin, MMP-9 and B-catenin expression were evaluated by immunofluorescence in vitro. (DAPI, blue; target protein, green). Furthermore, effects of the presence of PG on CSC-related proteins with (c) Flow cytometric analysis of CD44 and PD-L1 using TE2 cancer cells; and (d) The changes of CD44, ALDH1 and PD-L1 expression were evaluated by immunofluorescence in vitro. (DAPI, blue; target protein, green) 
(b) Invasion assay
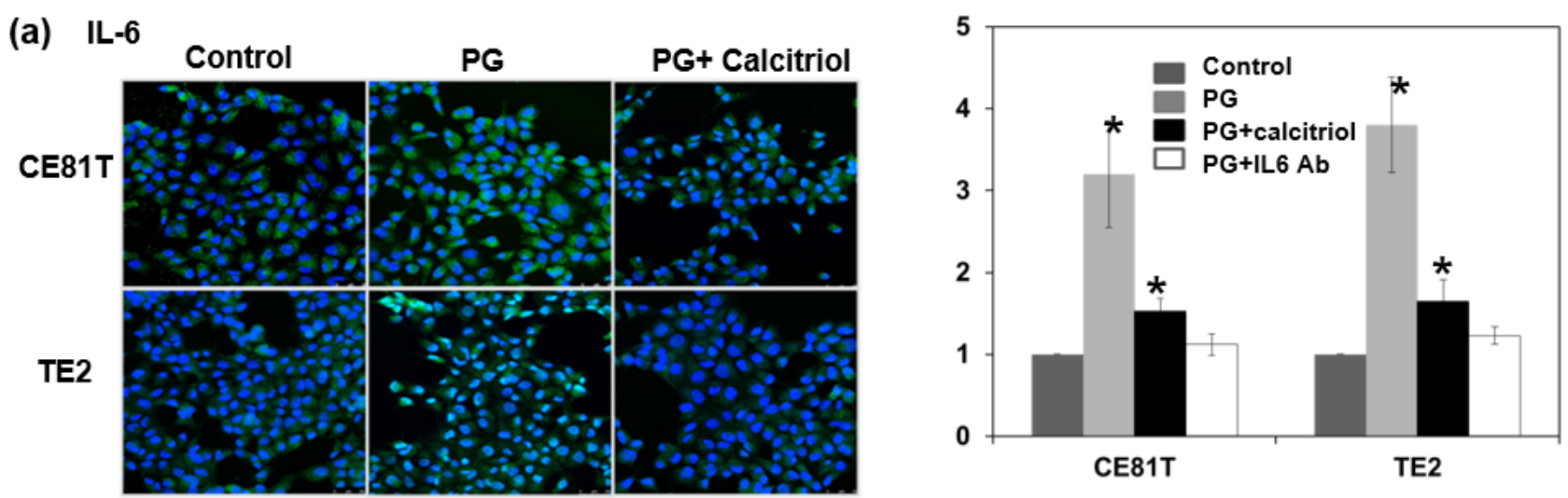

(c) B-catenin

(d)
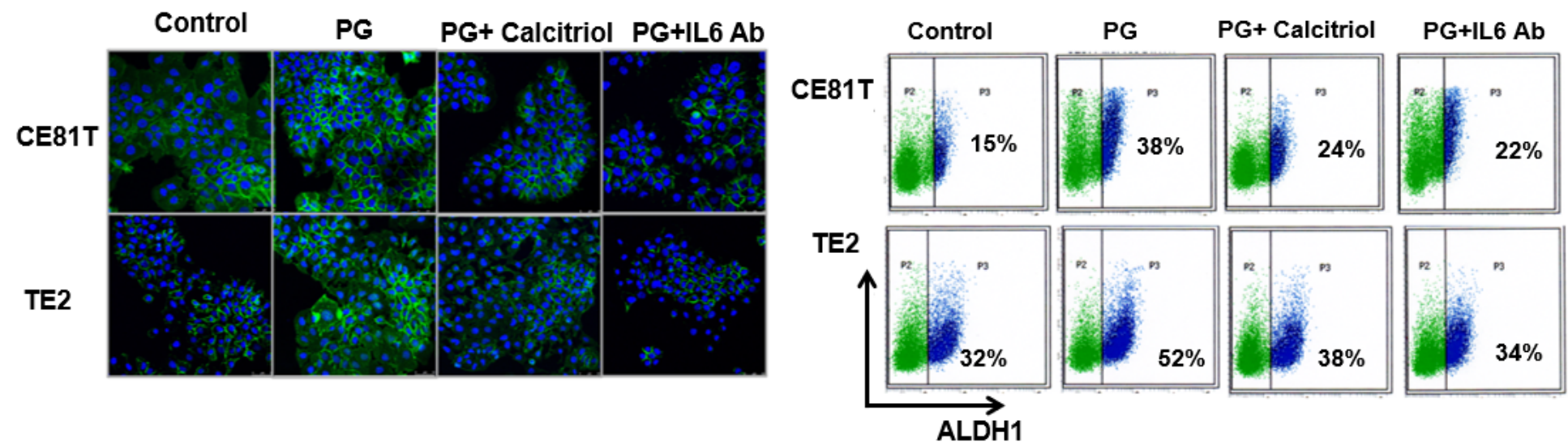

Figure 5

Effects of Calcitriol on IL-6/STAT3 signaling and tumor aggressiveness in PG-infected cells in vitro. Effects of Calcitriol treatment in PG-infected cancer cells on (a) the expression of IL-6 by immunofluorescence in vitro; (b) the invasive ability of esophageal cancer cells. The quantitative data ( $y$ axis shows the relative ratio, normalized to the number of invading control cancer cells) are shown, and (c) the EMT-associated proteins. The changes of B-catenin expression were evaluated by immunofluorescence in vitro. In addition, the expression of ALDH1 from cancer cells with or without calcitriol treatment and PG infection was evaluated by FACS in vitro. 
(a)

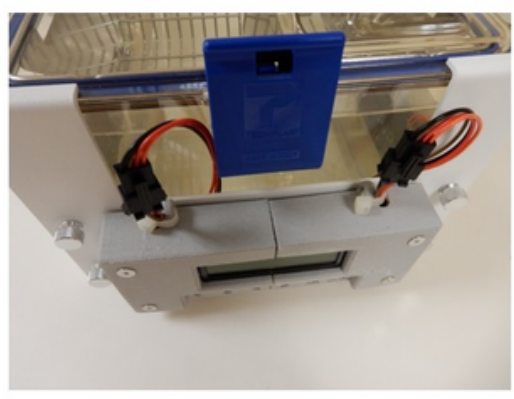

(b)

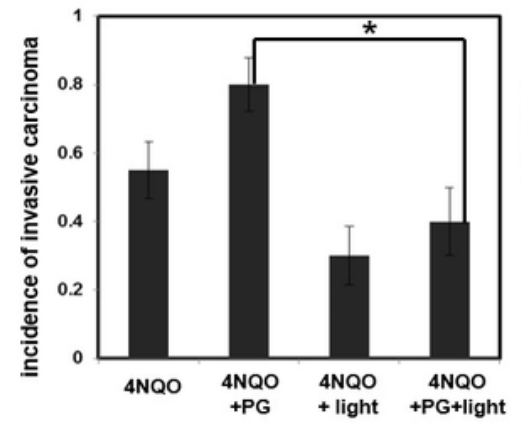

Vit D3 (pg/ml)

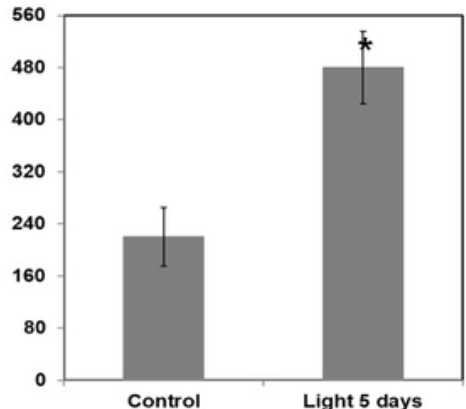

Control Light 5 days

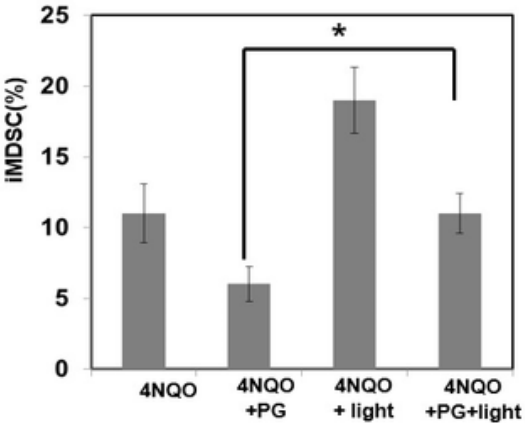

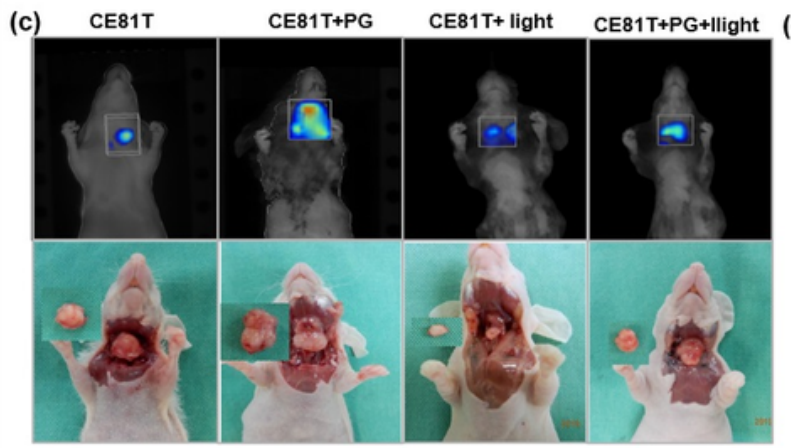

(e)
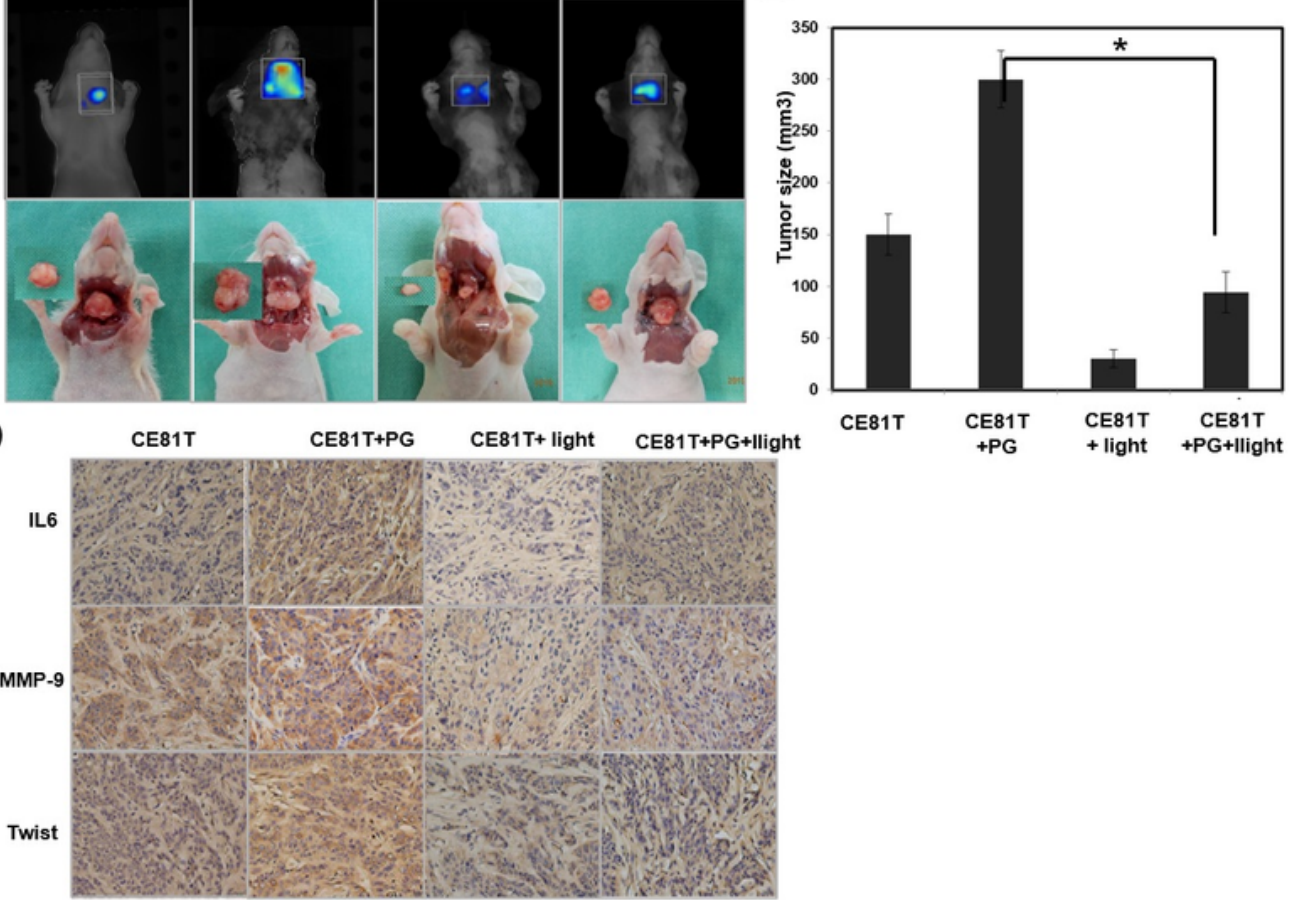

Figure 6

Effect of UVB-light treatment on the levels of calcitriol and tumor growth in animal tumor model The mice were received UVB irradiation using the designed device (a), associated the increased calcitriol in serum from C57/BL6 mice received UVB-light treatment or sham-irradiation for 5 days. (b) The quantitative data assessing the incidence of esophageal tumors, and the recruitment of MDSC from 4-NQO-treated mice with or without the presence of PG or UVB-light treatment are shown. Columns represent the means \pm 
SEM. *, $p<0.05$. Furthermore, (c) In xenograft tumor model, representative images of FMT examination and the gross lesions of orthotopic esophageal tumor in nude with or without the presence of PG or UVBlight treatment are shown. (d) The quantitative data assessing the orthotopic tumor size in nude mice are shown. Columns represent the means \pm SEM. *, p $<0.05$ (e) The changes of EMT-related protein and IL- 6 in tumor specimen evaluated by immunochemical staining in vivo. The results are shown by representative slides.

\section{Supplementary Files}

This is a list of supplementary files associated with this preprint. Click to download.

- Supplementarydata1.pdf

- Supplementarydata1.pdf 